\title{
A Survey on Authentication, Counterfeit Note Detection\& Automatic Printing System
}

\author{
Vivek D. Ugale ${ }^{1}$, Harsha Nyati ${ }^{2}$, Neeraj Shahane ${ }^{3}$, Harshada Bhalerao ${ }^{4}$ \\ Assistant Professor, Department of Electronics \& Telecommunication Engineering, SITRC, Nashik, India ${ }^{1}$ \\ Students, Department of Electronics \& Telecommunication Engineering, SITRC, Nashik, India ${ }^{2,3,4}$
}

\begin{abstract}
In every educational institute, government offices printing play a major role. Now a day, printing is easy task because of the different printing machines available in the market. Printing requires human efforts so the system is semi- automatic. Printing at public places where beneficiary has to pay for printing, is still vastly dependent on the operator i.e. manual help. Also it is difficult to print the pages during rush time period which causes further time delay. To avoid the manual help in paying charges for printing and delay in printing, we are designing the standalone automated printing system which will deliver prints to authenticated user without any manual help other than user himself/herself. Every task from taking printouts to paying the charges for the printing will be done without any help from operator. For security purpose and to avoid misuse of system, authentication is necessary part. To avoid counterfeiting of currency while depositing the money, fake currency detection module will be needed. In this paper we are reviewing the authentication, technique of currency detection and the automated printing system designed in the past.
\end{abstract}

Keywords: Authentication, automation, fake currency detection, printing, standalone.

\section{INTRODUCTION}

As this is the age of automation, almost every task in industries has been automated. Now-a-days there are many things where automation has brought radical changes. Be it a automobile manufacturing industry or a electronic chip producing industry or food industry, every industrial environment has been running on automation.

We often refer time as money, so it becomes inevitable to save the time by all possible means. In places such as shopping malls, wholesale and retail outlets, automation is incorporated for the automatic delivery of the products to the customers. Now a day's automated machines can be increasingly found to encroach the shops, which reduces human efforts require to search, count and deliver the products along with cash handling. The Automatic Teller Machine (ATM) is quintessential example of engineering principles to reduce human effort.

Mechatronics is the mixture of electronics engineering and mechanical engineering application and on that automated machines operate. The existing problem in printing machine is operator is required for printing the pages and taking the money as payment for printing. Every time people wentto printouts shop. Thus we are trying to make a standalone Automated Printing Machine, with the help of Mechatronics principles.

Paper is organized is as followed. Firstly, we are reviewing the authentication techniques, after that a brief discussion of research done on counterfeit currency detection given. Lastly survey of automated printing system is given. Concluding remarks are given in conclusion.

\section{LITERATURE SURVEY}

As the proposed standalone automated printing system contains authentication module, fake currency detection sensor and a mechatronics setup for depositing the money, in this Chapter firstly we are reviewing the authentication and fake Currency detection techniques and then giving the brief survey about the implemented automated printing system in the past.

\section{Authentication}

Authentication is a process of confirming an identity or an origin of a communication partner or a piece of information. Authentication makes it possible for an entity to verify that it really is interacting with those users and devices. Hence Misbehaving of devices and users is prevented by authentication.

Authentication is a vital part or our everyday life and present, for instance, when making phone calls, when using a wireless headset, when watching a pay television, when opening electronic locks in an office, or when doing transactions within Internet banks. So to identify the legitimate user for using the proposed system is a very necessary step.

Internet of things based on RFID (Radio Frequency Identification) has become focal point of attraction for industries and academia. But security is the main issue of IOT based on RFID.

Xiao Nie , XiongZhong proposed RFID system, for privacy protection and anti-interference, killing, Tag sleeping and Tag blocking methods introduced, in the communication process, the authentication based on HASH is the main method.[1] Tags, readers and antennas 
are the main parts of RFID system. Sometimes information leaked to unauthorized person using RFID tag for that encryption is needed. Receiver sends authentication that corrects tag is sent. In tag killing technique user put a kill command that disabled the tag so anyone cannot track that tag. But there is one drawback that RFID is not working here, for that tag sleeping technique is used to make the tag disabled when need not to be tracked i.e, temporarily on sleep mode. Fingerprint verification is an important user identification biometric technique.

The experiments done by Anil K. Jain et.al.reveal that this technique is good and secured on this data bases. Biometrics is a technology that identifies a person based on his physiological or behavioral characteristics. Fingerprint identification has medium universality, high uniqueness.

In automatic fingerprint identity authentication system there are four main design components: acquisition, representation (template), feature extraction, and matching. Inked and live scan these are the methods used for capturing image of fingerprint. In Live scan optical frustrated total internal reflection (FTIR) is the main concept. When on a glass prism user put his/her finger, ridges of the finger are in contact with the platen but the valleys of the finger are not. Representations based on the entire grey scale profile of a fingerprint image are prevalent among the verification systems using optical matching.

Brightness variation, image quality variations, scars are the factors affect to image quality. This system proposed and implemented by L. Hebbes and C. Chan uses a known mobile phone to decode an encrypted message transferred as a $2 \mathrm{D}$ barcode and read via a camera on the mobile device. [3] In this system mobile camera is used to read the encrypted message which is transferred as 2D barcode. 2D barcode generate onetime password on mobile phone which is random and difficult to remember. Data Matrix, PDF417, Encode, Semacode, and QR Code are the different 2D barcode types. QR codes used to track commercial applications. These contains information like as URLs, names, addresses, tele- phone numbers, e-mail addresses, etc. 2D barcodes and mobile camera used as visual channel for authentication.

Data exchange became easy using QR code with registered mobile phones. Same as for accessing webpage, for authentication user need to enter username then QR code displays user need to capture in camera. That encrypted information user enters as PIN then system generates 8-character code and that user enters as password.

\section{Counterfeit Currency Note Detection}

Counterfeit or fake notes are major problem occurring in cash transactions in a country like India. According to the survey conducted by RBI in 2013 shows that there is trillions of money in counterfeit notes in whole India. This is possible because as it has become very easier to print the fake notes with different latest tools due to advancement in technology.

Financial system of country is affected by the counterfeit currency. So identification of counterfeit and denomination is essential especially in money deposit machine. Detecting the fake notes is quite time consuming task so we cannot perform a task with human interference. For detection we need automated systems through which we can recognize the original currency.

\section{Fake currency detection}

Binod Prasad Yadavet. al. have proposed and implemented detection of fake notes using MATLAB and feature extraction with Hue Saturation Value (HSV) color space and other applications of image processing.[4] Manual testing of notes is time consuming and chances of tearing a note. For accuracy the characteristics should get matched is necessary.

In this with help of camera they are taking pictures of the note and analyzing it with the MATLAB Program. And check the note whether it is fake or genuine by checking parameters like See through register, water marking, optically variable ink, fluorescence, security thread, intaglio printing, latent image, micro lettering, identification mark.

Similar to the previous paper, Swami Gururaj $M$ and Naveen $\mathrm{J}$ too used basic image processing algorithm HSV conversion to identify the denomination and counterfeit of currency automatically using raspberry pi as a hardware platform.[5] Features like aspect ratio identification, dominant color, feature extraction and template matching, comparison with threshold values, Image acquisition, Image pre-processing, Image localization, Feature extraction, Template matching are the parameters to identify real currency The implemented system includes several steps like image capturing with camera, preprocessing of image and some algorithms to identify the original currency.

To check the originality of currency it is necessary to place note under web camera. As camera gets enabled by processor raspberry pi it will capture images of the note. Captured image will be send to processor, for identification of counterfeit and denomination of currency processor will process the image. MATLAB is used for writing a code and algorithm. Raspberry pi supports execution of simulation model so need to convert code to the simulation model. For counterfeit currency detection, KameshSanthanamet.al.illustrated technique depending on 2 mechanisms first one being the Ultra Violet (UV) detection using Lab View and other one is using the polarization of light when passed through the currency. Result is considered positive if both the outputs are positive.[6] One of the methods is UV detection.

It is principle which detects inks which are visible under UV light. Automation is introduced using (NI-IMAQ). Indian currency and counterfeit currency, both are coated with dyes which are visible in UV light so to check the originality it requires human efforts. Using National 
Instruments-Image Acquisition (NI-IMAQ) they have made this process automated.

They have taken original image of different currency from various angles and stored those images in a computer. Currency is captured by HD camera and sent to IMAQ LABVIEW. And the current image is get compared with original currency in database, if currency is real status glows green else red. By measuring the polarization properties of cellulose we can extend property of currency. As the currency is translucent, it is necessary to use monochromatic laser to obtain a beam for the photo detector to detect. The polarization compensator is used to measure the circular and elliptical polarization. And this is achieved by using a servo motor controlled by the micro controller. Depending upon the data fed into the micro controller, the servo motor makes its movement and we get the appropriate polarization since the light coming out of a translucent material is of less intensity, we use a highly sensitive photo detector amplifier array to measure the intensity.

Since maximum intensity is obtained when the plane of polarization is parallel to the plane of vibration, this method is used to determine the genuineness of the currency. In this paper, S.Sukhumaret.al, instead of manual system for rationing, automated system based on smart card is proposed and implemented.[7] There are three interfaces, touch screen, billing printer and GSM module, these devices are interfaced to microcontroller and connected to the database of the central government.

The user is provided with RFID cards, those who wants to avail the service has to swipe the same, after swiping authentication will be done using the ADHAAR card and user finger print, once user is authenticated system will update the user information on touch screen. After the authentication, input regarding the product which user wants to purchase will be given on the touch screen.

After input given by user, system will check the amount in account of user if amount is sufficient for the transaction, then grain will come out from the machine output and the amount will be deducted from the bank account. As the government database is linked with the proposed system, using GSM module system will send the updated information about purchased product, account balance etc. To the customer and government As system is using the UID (unique identification) number i.e.

ADHAAR number the government of India has given which includes all information such as age, no of members in family, finger print off all members in family, bank account information address contact numbers etc. for every citizen of India. So it provides additional facet for authentication of user along with fingerprint If overcrowded by customer, charges would be more than prescribed rates, Cost effective, Time saving, system will help to maintain the data, safe secure and efficient as authentication of user needs smart card, password and fingerprint of legitimate user. This is system is implemented by M.Bhuvaneswariet.al ,each person is allotted with a smart card which has a RFID on it.[8] The
RFID is linked with the bank account of the user. Once RFID is scanned at the ticket vending machine a list of destinations and their respective bus timing is displayed on the touchscreen user have to select the destination and busses for travel. User has to again scan the smart card at the bus door in order to open it. an alcohol sensor is also present at the door it will not allow the drunken person to enter in the bus.

With the help of voice GPS passengers can identify the upcoming locations and is beneficial to blind people to use voice GPS This system can completely eliminate the old paper ticketing method. Ticket friendly method give access to transfer ticket from 1 person to another and can reduce money issues as the balance is deducted directly from bank account.

Smart card or touch and go cards can store the travel information and proof of identity of user in memory or microprocessor for safe and convenient travel. After the RFID is scanned the pay mode terms are displayed which contains amount in the account. The user can recharge these smart card as per their requirement. After scanning RFID, for transmission of data of transport facilities Zig Bee Is used on huge display Real time bus information system (RTBIS) may also employed in this system that uses satellite technology to predict the time of bus which will arrive at a stop.

RTBIS display data like ticket cost, seats availability and location to the bus operator by using Zigbee he can identify the location where the passengers preferred. After the data given by the user a security code is printed on the RFID card. Zig Bee is important as inter connection of wireless sensor vehicles and infrastructure. RohanBhirangi, SmitaBhoir proposed a system in which particular course related questions are stored. This hierarchy restricts access to user.

Duplication of paper is prohibited due to security mechanism. Any educational institute can modify the contents for particular subject, semester and syllabus content. Now this feature allows the institute to ensure security and avoids repetition of questions. This system aims to provide fast operations, data storage and high security for all its tasks.

The system is entirely governed through the login screen. Only authenticated user can log into system using his credentials. On successful login, various systems are available to the user depending on his/her role in the hierarchy.

A data entry user (or the admin) can enter various data and customize the system according to the organization. The administrator has data entry option and Paper generation that is only accessed by paper generator.

In this system illustrated by Kamalanathan.Pet.al.mechatronic is used for vending machine which dispenses snacks cigarettes alcohol etc. Vending machine are mostly used examination seasons near educational institutions and almost everlasting demand near the government offices. It includes input 
unit, control unit and output unit.

The processing unit includes the sensing of the reception of the genuine currency coin through the coin inlet, deciding the number of paper to be delivered, instructing the electrical motor to deliver exactly the number of paper, to be delivered. The mechanical part of the system includes the working mechanism. The mechanical components include the rollers, shaft, bearings, gears etc.

The electrical system contains motors, power supply with transformer in the automatic paper vending machine. A currency detector detects the currency is valid or not. a process verify the currency is valid or not by various tests. The papers are placed such that the roller makes the contact with the first paper and it is rolled in when the electric impulse is passed.

Then validation of coin is verified by sensor. if the coin is valid then the processor starts the dc motor the roller pass out the 1st paper as the above steps are processed Faster delivery of papers to the customers would be possible during peak time and the unnecessary crowd near the stationery shops near the educational institutions, during examination season would be avoided.

This technique proposed by Mohd. ArifSiddique and Dr.Amit Kumar Awasthi are used to give service to customer to make DD by ATM.[11] This technique is used to overcome the problems faced by customers that DD cannot prepare on time such as in night or off days. Ebanking is used for this technique. This DD making technique developed by making small changes in ATM. Need to replace the keypad such as in mobile phones.so users can enter name, account number, branch just like writing SMS in phone. Making minor changes in ATM, hardware and software cost could not increase.

In existing ATM transaction slip printer, added a new printer that can print DD as well. In this system there is paper selector which having 2 roller set, one to forward paper of transaction slip printer and another to forward paper of DD. This system suggests new layout for DD. Change layout of DD from landscape to portrait.

So that DD printed by the same printer of transaction slip and no need of special slot. Signing authority is to branch manager where ATM is associated. This technique gives strength to the anywhere banking. Anytime DD making is possible. Also customer can get DD at the spot, better than online service.

\section{III.CONCLUSION}

After reviewing the various papers based on user authentication, currency detection and some of the previously implemented printing system analogous to what we are considering to design, we can conclude that biometric authentication in most secure method for user authentication. As for the detecting the counterfeit currency notes, different features of the currency note can be processed using HSV algorithms using MATLAB is most effective method. As we are considering making automated printing system, these above mentioned methods can be used for building the standalone automated printing system.

\section{REFERENCES}

[1] Xiao Nie, XiongZhong," Security in the Internet of Things Based on RFID: Issues and Current Countermeasures", Proceedings of the 2nd International Conference on Computer Science and Electronics Engineering (ICCSEE 2013)

[2] Anil K. Jain et.al.,"An Identity-Authentication System using Fingerprints", Proceedings Of The Ieee, Vol. 85, No. 9, September 1997

[3] L. Hebbes and C. Chan ,"2-Factor Authentication with 2D Barcodes", Proceedings of the Fifth International Symposium on Human Aspects of Information Security \& Assurance (HAISA 2011)

[4] Binod Prasad Yadav, C. S. Patil, R. R. Karhe, P.H Patil, "An automatic recognition of fake Indian paper currency note using MATLAB," International Journal of Engineering Science and Innovative Technology (IJESIT) Volume 3, Issue 4, July 2014

[5] Swami Gururaj M., Naveen J., "Identification of Counterfeit currency and denomination using Raspberry pi," Nitte Conference on Advances in Electrical Engineering NCAEE-2016, Vol. 4, Special Issue 2, April 2016

[6] KameshSanthanam et.al, "Counterfeit Currency Detection Technique using Image Processing, Polarization Principle and Holographic Technique," Fifth International Conference on Computational Intelligence, Modelling and Simulation,2013

[7] S.Sukhumar et.al, "Automatic Rationing System Using Embedded System Technology," International Journal of Innovative Research in Electrical, Electronics, Instrumentation and Control Engineering Vol. 1, Issue 8, November 2013

[8] M.Bhuvaneswari et.al," Embedded System Based Automatic Ticket Vending Machine for Modern Transport System", International Journal of Advanced Research in Computer and Communication Engineering Vol. 2, Issue 11, November 2013.

[9] RohanBhirangi, SmitaBhoir, "Automated Question Paper Generation System," International Journal of Emerging Research in Management \& Technology, (Volume-5, Issue-4), April 2016.

[10] Kamalanathan.P, Irshath Ahmed. R, Mohamed Aamir. M, Kalaiselvan. P, “Automatic Paper Vending Machine,” International Journal of Science, Engineering and Technology Research (IJSETR), Volume 4, Issue 4, April 2015.

[11] Mohd. ArifSiddique and Dr.Amit Kumar Awasthi, "An Advance ATM Machine Service: Making Demand Draft through ATM Machine," 5th IEEE International Conference on Advanced Computing \& Communication Technologies [ICACCT-2011] ISBN 81-87885-03-3. 